Relations industrielles

Industrial Relations

\title{
The Challenge to Management Control, by John Storey, Brookfield, Vermont, Renouf USA, 1981, 192 pp., ISBN \\ 0-09-145941-9.
}

\section{Jacques Bélanger}

Volume 37, numéro 2, 1982

URI : https://id.erudit.org/iderudit/029268ar

DOI : https://doi.org/10.7202/029268ar

Aller au sommaire du numéro

Éditeur(s)

Département des relations industrielles de l'Université Laval

ISSN

0034-379X (imprimé)

1703-8138 (numérique)

Découvrir la revue

Citer ce compte rendu

Bélanger, J. (1982). Compte rendu de [The Challenge to Management Control, by John Storey, Brookfield, Vermont, Renouf USA, 1981, 192 pp., ISBN

0-09-145941-9.] Relations industrielles / Industrial Relations, 37(2), 455-457.

https://doi.org/10.7202/029268ar

Tous droits réservés @ Département des relations industrielles de l'Université Laval, 1982
Ce document est protégé par la loi sur le droit d'auteur. L'utilisation des services d’Érudit (y compris la reproduction) est assujettie à sa politique d'utilisation que vous pouvez consulter en ligne.

https://apropos.erudit.org/fr/usagers/politique-dutilisation/ 
ge; il doit même, sous peine de poursuites en dommages encourager activement ses membres à la respecter...

Cette transformation, en fait, graduelle mais globale, du droit du travail, son passage d'une orientation collectiviste, qui laisse place au rôle dynamique des conflits collectifs, à une fonction d'instrument d'assujettissement de l'action collective aux objectifs de l'État, résulte de l'influence combinée de différentes mutations sociales, économiques et politiques. L'introduction (ou partie première) du présent volume permet au lecteur d'évaluer ces facteurs à l'aide des riches données qu'elle contient. Ces données se rapportent à des sujets tels l'évolution du chômage, la progression des effectifs syndicaux, la fréquence du recours à la grève. On y expose aussi les principaux événements qui surviennent dans le cheminement malheureux de la République de Weimar.

Chacun des trois articles du professeur Kahn-Freund jettent eux-mêmes la lumière pénétrante de son analyse sur la transformation concomitante du système juridique. Le premier de ces textes, mais aussi le plus récent, ne date pas de cette période où l'auteur était encore activement engagé dans la magistrature du travail dans son pays d'origine, contrairement aux deux autres qui le suivent dans l'ordre de présentation du volume. Il s'agit d'un essai biographique sur le professeur Hugo Sinzheimer, qui vécut de 1875 à 1945 et en qui l'on peut voir l'un des grands fondateurs, à la fois d'un point de vue législatif et doctrinal, du droit du travail allemand. Notons en particulier de la part de celui qui allait accentuer, durant son exil hollandais débutant en 1933, sa réflexion sociologique sur le droit, des vues sur la fiction libérale du contrat individuel de travail ( $p$. 102). Les deux articles suivants de KahnFreund datent respectivement de 1931 et 1932. Dans "The Social Ideal of the Reich Labour Court", ce dernier s'insurge, courageusement dans les circonstances, contre la subtile - mais gratuite, d'un point de vue juridique - importation de concepts et attitudes fascinantes dans la jurisprudence du travail, et plaide - quelles que soient en définition les valeurs politiques retenues - en faveur de la nécessité de procédés transparents et de l'indépendance judiciaire. La conclusion (p. 155) vaut d'être reproduite intégralement:

"There are many roads which lead to
dictatorship. However, the most likely
road to fascism in our social condi-
tions is not through force, but through
the veneration of peace and order, of
discipline and welfare provision, and
above all through the ideological inte-
gration of the organisations of strug-
gle into a hierarchically - ordered na-
tional community."

Enfin, "The Changing Function of Labour Law", initialement publié dans Archiv für Socialwissenschaft und Sozialpolitik, est la synthèse brillante de cette transformation graduelle du droit du travail - non tant les textes, mais surtout leur interprétation judiciaire et leur mise en oeuvre administrative - durant la République de Weimar et dont quelques traits seulement ont été évoqués dans les lignes ci-avant.

Cet ensemble documentaire et analytique que constitue Labour Law and Politics in the Weimar Republic est manifestement d'un grand apport scientifique. Par ailleurs, les trois textes du professeur Kahn-Freund n'auraient-ils pas aussi, plus généralement, une troublante portée prémonitoire?

Pierre VERGE

Université Laval

The Challenge to Management Control, by John Storey, Brookfield, Vermont, Renouf U.S.A., 1981, 192 pp., ISBN 0-09-145941-9.

Ce volume traite de la portée des limites imposées aux droits de la direction par les travailleurs et leurs syndicats dans l'industrie britannique. Il s'inscrit dans la foulée d'une thèse de doctorat présentée par l'auteur à l'University of Lancaster en 1974. Le sujet 
traité, celui du rapport entre les prérogatives patronales et la résistance syndicale et ouvrière, est certes des plus fondamentaux dans le champ des relations industrielles.

Bien que l'auteur ne le présente pas comme tel, on identifie deux parties bien distinctes dans ce volume. En bref, les quatre premiers chapitres consistent en une revue de la littérature et une discussion d'ordre analytique sur les prérogatives et le contrôle managérial, alors que dans la seconde partie (à partir de la page 108), l'auteur présente les résultats de sa recherche empirique. Il nous semble approprié de suivre cette distinction pour les fins de cette recension.

En première partie, l'auteur souligne avec raison que dans la période contemporaine, la question des prérogatives patronales est vraiment soulevée lorsque les travailleurs s'efforcent d'exercer un contrôle relatif sur la façon dont leur force de travail est utilisée dans le processus de production. Les travailleurs et leurs syndicats touchent alors au coeur même de la fonction managériale, laquelle est centrée avant tout sur la fonction de contrôle. L'auteur soutient que la nécessité de ce contrôle managérial sur la force de travail "emerges from the need to surmount two kinds of uncertainty with regard to the labour factor" (p. 57). La première consiste en la difficulté d'atteindre la réalisation optimale de ce potentiel que constitue la force de travail (en somme la distinction entre force de travail et travail réalisé). La seconde réfère à la résistance collective des travailleurs à la subordination.

Dans cette discussion, Storey centre aussi l'analyse sur le concept de prérogatives patronales qui, selon lui, avait jusqu'alors été négligé dans la littérature britannique. Ce concept est défini comme étant "the residue of discretionary powers of decision left to management when the regulative impacts of law and collective agreements have been subtracted" (p. 41). L'auteur ne parvient pas à nous convaincre que l'on puisse marquer un progrès sur le plan analytique à partir de cette notion. En bref, il s'agit toujours de cette question de la «frontière du contrôle», objet de la thèse de Goodrich publiée en 1920. La première partie de l'ouvrage de Storey ne représente donc pas à notre avis un progrès significatif sur le plan analytique.

Après avoir argumenté à l'effet que le véritable défi au contrôle managérial ne provient pas de l'État, ni de la représentation nationale des syndicats, l'auteur passe ensuite à la partie empirique. L'attention du chercheur est alors centrée sur la portée de cette érosion des droits de la direction, suite à la décentralisation des relations du travail vers le niveau de l'établissement. Il lui est possible d'étudier la tendance au cours des années 1970, à partir de la recherche effectuée au début de la décade et d'un suivi dans les mêmes établissements en 1978. La recherche couvre cinq secteurs d'activité: les brasseries, le transport public, le textile, les fibres synthétiques et l'industrie mécanique. La recherche consiste d'abord en des études de cas dans six établissements, puis en une enquête postale par questionnaire effectuée en 1971 et en 1978 auprès de représentants de la direction (96 questionnaires valides en 1978) et de délégués d'atelier (132 questionnaires) travaillant dans ces mêmes secteurs d'activité.

Les données recueillies indiquent d'abord que si l'institutionnalisation des relations du travail au niveau de l'établissement est plus avancée dans l'industrie mécanique, le progrès en ce sens a été substantiel dans les autres industries, au cours des années 1970. Quel fut l'impact de cette formalisation sur la portée de la négociation? À partir d'une grille de 25 matières (autres que la rémunération), les réponses à l'enquête postale permettent de conclure à un élargissement significatif de la portée de la négociation dans toutes les industries. Et les résultats montrent que les répondants ont effectivement perçu un tel accroissement du domaine négociable. Les représentants des deux parties s'entendent aussi assez bien sur l'identification des matières faisant le plus fréquemment l'objet de négociation. Les matières suivantes sont négociées dans plus de la moitié des établissements: les quarts de travail, les niveaux d'emploi, le temps supplémentaire, les mutations, la disci- 
pline, les cadences de travail et le contenu de l'emploi. Par ailleurs, la portée de la négociation est plus grande dans l'industrie mécanique, suivie du secteur du transport public; le secteur du textile est de loin celui où on négocie sur la gamme la plus limitée de sujets.

L'auteur conclut avec cette formulation du dilemme dans lequel se trouvent les dirigeants d'entreprise britanniques soucieux de préserver leur contrôle: "They can attempt to incorporate the shop stewards' organization, and in the process bolster it so that it may exercise some control over its member (and this carries the inherent risk that the fortified creature may also bite its master's hand). Or, shrinking from the risk, the alternative response is to stand on the doctrine of employers' rights and refuse to treat with the internal challenge. This option requires rigorous and forceful supervision..." (p. 177). Évidemment, la stratégie patronale dépendra d'abord de l'évaluation du rapport de force dans chaque établissement, les conditions variant beaucoup à l'intérieur d'un même secteur d'activité. Mais elle pourrait aussi dépendre de la mesure dans laquelle la régulation conjointe sur des matières comme celles identifiées plus haut pose de sérieux problèmes quant à l'accomplissement de la fonction première de l'entreprise, soit la production. (Cette question fondamentale, mais aussi très difficile, est soulevée très brièvement en pages 164-5.) Les relations du travail étant une fonction dérivée de la fonction de production, le management ne devrait-il pas réagir en fonction des contraintes imposées à la production? Dans quelle mesure le contrôle managérial s'est-il affaibli à cet égard depuis dix ans?

Dans ce volume, les résultats les plus substantiels proviennent de l'enquête postale et cette méthode peut difficilement nous faire progresser sur la question soulevée ci-haut puisqu'elle ne favorise pas l'étude du procès de travail et du rapport de production. (L'apport des six études de cas est faible à cet égard.) Cela explique aussi une autre limite de cet ouvrage: il nous informe relativement bien sur la portée de l'influence ouvrière mais très peu sur le degré relatif de contrôle exercé par ceux-ci.

L'auteur serait toutefois justifié de nous rappeler que seule l'observation en profondeur dans un ou quelques établissements est susceptible de générer ce genre d'information qualitative sur le degré de contrôle. Et il ne manque pas de souligner dans ce volume les limites propres à ce genre d'étude de cas dont on ne peut postuler la représentativité. En ce sens, les contributions complémentaires de l'observation systématique et de l'enquête sur échantillon sont souhaitables. L'ouvrage présenté ici correspond davantage au second type de contribution. En ce sens, il faut souligner l'apport significatif de cette recherche empirique qu'il faut recommander à ceux qui étudient les relations du travail. C'est le fruit d'une recherche qui s'étend sur une période d'une dizaine d'années et qui a le mérite de dépasser le cadre de l'industrie mécanique, laquelle a jusqu'ici canalisé une grande proportion de la recherche sur les lieux de travail en Grande-Bretagne.

\section{Jacques BÉLANGER}

Université Laval

\section{Organization Theory: A Macro Perspective} for Management, $\left(2^{\mathrm{e}}\right.$ ed. $)$, by John $\mathrm{H}$. Jackson and Cyrill P. Morgan, Englewood Cliffs, Prentice-Hall Inc., 1982, 413 pp., ISBN 013-641415-X.

Cet ouvrage s'adresse avant tout aux étudiants en sciences de l'administration, en sciences de l'éducation, en sociologie, en science politique et en relations industrielles. De fait, on retrouve dans les répertoires de cours offerts par ces facultés ou départements un enseignement qui porte plus précisément sur la structure des organisations et sur leurs processus de survivre et de développement. La présentation de la matière correspond à celle qu'on retrouve dans tout bon syllabus de cours sur le sujet. D'abord, l'importance des organisations de travail dans la société actuelle; l'évolution des connaissances dans le domaine... Ensuite, une revue rapide des au- 